\title{
REVIEW ARTICLE \\ Reflection paper on MRSA in food-producing and companion animals: epidemiology and control options for human and animal health
}

B. CATRY ${ }^{1 *}$, E. VAN DUIJKEREN ${ }^{2}$, M. C. POMBA ${ }^{3}$, C. GREKO ${ }^{4}$, M. A. MORENO ${ }^{5}$, S. PYÖRÄLÄ ${ }^{6}$, M. RUŽAUSKAS ${ }^{7}$, P. SANDERS ${ }^{8}$, E. J. THRELFALL ${ }^{9}$, F. UNGEMACH ${ }^{10}$, K. TÖRNEKE ${ }^{11}$, C. MUŇOZ-MADERO ${ }^{12}$ AND J. TORREN-EDO ${ }^{12}$ on behalf of the Scientific Advisory Group on Antimicrobials (SAGAM)

${ }^{1}$ Scientific Institute of Public Health, Epidemiology Unit, Brussels, Belgium; ${ }^{2}$ Faculty of Veterinary Medicine, Utrecht University, The Netherlands, ${ }^{3}$ Faculty of Veterinary Medicine, Technical University of Lisbon, Portugal; ${ }^{4}$ National Veterinary Institute, Uppsala, Sweden $;{ }^{5}$ Universidad Complutense, Madrid, Spain;

${ }^{6}$ Faculty of Veterinary Medicine, Department of Production Animal Medicine, University of Helsinki, Finland;

${ }^{7}$ Veterinary Institute of Lithuanian Veterinary Academy, Kaisiadorys, Lithuania ; ${ }^{8}$ Agence Française de Sécurité Sanitaires des aliments (AFSSA), Laboratoire d'Etudes et de Recherches sur les Médicaments Vétérinaires et les Désinfectants, Fougères, France; ${ }^{9}$ Gastrointestinal Emerging and Zoonotic Infections Department, Health Protection Agency, London, UK; ${ }^{10}$ Veterinärmedizinische Fakultät, Institut für Pharmakologie, Pharmazie und Toxikologie, Universität Leipzig, Germany; ${ }^{11}$ Läkemedelsverket, Uppsala, Sweden; ${ }^{12}$ European Medicines Agency (EMA), London, UK

(Accepted 15 December 2009; first published online 9 February 2010)

\section{SUMMARY}

The scope of this reflection paper was to review the latest research on the risk of MRSA infection and colonization in animals. Attention focused on occurrence, risk factors for colonization and infection, and human contact hazard for livestock, horses, and companion animals. Whereas the clonal relationship between MRSA strains of CC398 is straightforward in livestock this is less obvious in horses. Small companion animals typically share MRSA strains that seem to exchange with a human reservoir. Management and therapeutic options have been suggested for livestock, horses, companion animals, as well as instructions on safety measures for persons in contact with animals. Conclusions were drawn with emphasis on future research activities, especially to confirm the apparent evolution of the organism and to demonstrate efficiency of control strategies.

Key words: CC398, companion animals, horses, livestock, MRSA, Staphylococcus aureus, ST398.

\section{INTRODUCTION}

An increased number of reports on methicillinresistant Staphylococcus aureus (MRSA) in livestock (in particular in swine) have been recently published. MRSA has also been found in companion animals

* Author for correspondence: Dr B. Catry, Scientific Institute of Public Health, Juliette Wytsmanstraat 14, 1050 Brussels, Belgium. (Email: boudewijn.catry@iph.fgov.be) and horses, and transmission between humans and colonized animals has been reported (reviewed in $[1,2])$. This apparent extension of the host range of MRSA to different animal species demands a critical review on the factors associated with this emerging zoonotic bacterium from both the veterinary and public health point of view.

The scope of the present reflection paper is to review the latest research on the risk of MRSA infection 
and colonization in animals in order to provide a preliminary risk profile for animal use of antimicrobials in relation to this risk. The document considers all food-producing and companion animal species. The major public health significance is focused on direct contact with living animals and not on animalderived food products.

The specific aims of the document are: $(a)$ to assess the impact of the use of antimicrobials in livestock and companion animals on the risk of colonization or infection with MRSA, and (b) to provide advice on management options for animals related to the issue. In addition, the document identifies those areas where the available information is absent or too small to allow reflections, and indicates areas for future research and data collection to focus on.

\section{STAPHYLOCOCCUS AUREUS}

$S$. aureus causes a wide range of severe and economically important diseases in human and veterinary medicine $[1,3]$. The bacterium is a colonizer of the skin and mucosae from which it can invade multiple organs. In livestock $S$. aureus is an important cause of mastitis, skin and soft tissue infections (SSTI) and to lesser extent infections of the locomotory system. Surgical site infections (SSI) in which $S$. aureus is isolated have been increasingly reported in small companion animals and horses [4].

In humans, prevalence of $S$. aureus infection varies widely between European member states, among hospitals and inside hospitals. The reasons for the difference are probably due to the level of screening, isolation and monitoring of patients and staff in hospitals, with the Dutch having the most pro-active system over the last decades. There is a shortage of quality data investigating infection and/or carriage rates in the community, but occurrence appears to vary substantially with geographical location [5].

\section{Human invasiveness}

$S$. aureus is by far the most important pathogen in SSTI including SSIs. Life-threatening nosocomial infections such as pneumonia and septicaemia also occur. Treatment of invasive infections largely relies on antimicrobial agents (antimicrobials), in this respect penicillinase-stable $\beta$-lactams (isoxazolylpenicillins, cephalosporins) are of utmost importance for human medicine. Resistance to these agents drastically limits therapeutic options and is a worldwide emerging problem [6]. It should be noted that in this document the applied definition of an antimicrobial does not include commonly used local antiseptics and disinfectants, e.g. chlorhexidine, alcohol, or soaps.

$S$. aureus can also produce toxins associated with food intoxications. Other rare but severe syndromes including toxic shock syndrome (TSS) have also been documented [7].

\section{Epidemiological definitions of MRSA groups}

$S$. aureus is intrinsically susceptible to $\beta$-lactam agents that inhibit cell-wall formation due to binding with proteins involved in the formation of peptidoglycan, as previously reviewed [8]. The mechanism of resistance to penicillinase-stable $\beta$-lactams including methicillin, isoxazolylpenicillins and cephalosporins in $S$. aureus (MRSA) is an altered target site due to an acquired penicillin-binding protein (PBP2a, also called PBP2') encoded by the gene mecA.

Different types of MRSA may be distinguished based on epidemiological groupings. This can be a simplistic approach since in some cases strains of MRSA have spread between the groups [7]. Thus it might be difficult to determine which epidemiological pattern a certain MRSA strain is associated with. The grouping is of relevance for the remainder of this document although only MRSA-related to animals will be discussed. It should be noted that virulence may differ between strains within groups and that toxin-producing strains may be found in any of the groups.

Hospital-associated MRSA (HA-MRSA). These have been known as nosocomial pathogens for decades. MRSA are regarded as HA-MRSA when infections caused by them are likely to be acquired in healthcare settings when they emerge at least $48 \mathrm{~h}$ after admission in patients having particular risk factors such as prolonged hospital stay, care in intensive-care units (ICUs), prolonged antibiotic treatment, surgical interventions, and/or close contact with MRSApositive individuals [9].

Healthcare-associated community MRSA (HCAMRSA). These are associated with outpatients with MRSA infection/colonization and previous hospitalization, such as residence in a nursing home, receiving home nursing, attending centres for dialysis and/or centres for diabetes were MRSA of hospital origin has been introduced [10]. 
Community-associated MRSA (CA-MRSA). These emerge in the community; affected patients lack the above-mentioned risk factors. Close contact in sport settings, schools, day-care centres, military settings and prisons, are considered risk factors.

Livestock-associated-MRSA (LA-MRSA). These refer mainly to the clonal spread of a certain MRSA strain (ST398, see below) that colonizes different food animal species (including horses) and may cause infections in humans.

Companion animals and horses may be colonized with a variety of strains due to their close contact with humans. Thus, these species may act as carriers of MRSA originating from humans (a so-called 'humanosis') [7].

\section{Emergence in animals}

During the period 1970-2000, MRSA has been sporadically isolated from animals, in particular cows, small companion animals, and horses. With the exception of some equine isolates, the nature of these cases suggested a human origin and no epidemics have been reported [1]. Thus, until the end of the 20th century both the scientific community and policy makers were convinced that animal husbandry was of little relevance to MRSA causing diseases in humans, but was rather a problem solely based on the antimicrobial use in human medicine [11]. The situation has now changed, with an increased number of reports on MRSA in livestock, especially swine and veal calves. MRSA has also been reported in companion animals and horses, as well as transmission between humans and animals. This has recently been reviewed [1]. Sometimes distinct animal specific-lineages such as LA-MRSA have been involved [12, 13], but on many occasions human-associated MRSA genotypes have been identified [14]. A note of caution in assessing the impact of 'animal' MRSA in human infections is that the increased awareness of such organisms is likely to have influenced the concentration of studies dealing with MRSA in animals. Interpretation and comparisons over time and between studies should be carefully performed since different procedures and approaches (study design) may have influenced prevalences.

\section{Zoonotic concerns}

Persons in direct contact with MRSA-positive animals have been shown to have an increased risk of carrying the same MRSA strains as the animals. This has been observed in small-animal healthcare, equine hospitals and livestock environments [7, 14]. Severe manifestations of LA-MRSA in humans have been documented, including a recent outbreak in a Dutch hospital $[15,16]$. This emerging phenomenon represents a hazard that demands a bilateral management approach, taking both animals and humans as potential sources of MRSA.

\section{Molecular typing}

The mecA gene is located on the mobile staphylococcal chromosomal cassette (SCCmec), and six major types have been found (I-VI). SCCmec types I-III are the most common in HA-MRSA [6]. These strains often carry additional plasmids or transposons enhancing the spread of resistance to two or more unrelated classes of antimicrobials (multiresistant). CA-MRSA typically harbour the smaller and possibly more mobile SCCmec type IV, and also type V, while multiresistance is less common. These strains often carry an exotoxin Panton-Valentine leukocidin (PVL) toxin; the pathogenicity of this toxin is still under debate [17]. HA- and CA-MRSA cannot be strictly separated because genotypic CA-MRSA are reported with increasing frequency in the healthcare environment $[6,18]$. LA-MRSA carry SCCmec types IV or V.

In addition to SCCmec typing which is relatively new and was discussed above, two important molecular-typing techniques for differentiation of MRSA are pulsed-field gel electrophoresis (PFGE) and multilocus sequence typing (MLST). In contrast to MLST, PFGE has the disadvantage of considerable variation of results between laboratories. MLST and PFGE can assist in ascertaining if strains are clonally related, but not all strains are typable with these methods. For example the typical LAMRSA strain which belongs to sequence type (ST)398 resists digestion by the $S m a$ I restriction enzyme by traditional PFGE and results are therefore not interpretable [19]. Some reports call these strains untypable or non-typable MRSA (UT or NT-MRSA). Recent evolutions in the genome can alter the MLST profiles, and strains approaching a certain type are defined as belonging to a clonal complex (CC). Spa typing is an upcoming important genotyping method which can differentiate strains that are indistinguishable by PFGE or MLST. ST398 belongs to CC398, and examples of spa types found are 
t108, t011, t034 [19]. The aforementioned and other techniques are under evaluation to increase the discriminative power especially with regard to MRSA ST398 [20].

Throughout this document and unless otherwise specified, LA-MRSA is the preferred term used as a synonym for UT-MRSA, NT-MRSA, MRSA ST398, and MRSA CC398.

The term colonization or carriage as used in the document refers to an individual person or animal that tests positive for MRSA in swabs from nares or throat (or other body sites) due to multiplication and settlement of MRSA not causing clinical symptoms [5].

\section{EPIDEMIOLOGY AND ECOLOGY OF MRSA}

\section{Occurrence in livestock}

The first report of MRSA in livestock was a case of bovine mastitis in Belgium [21]. Although moleculartyping methods were not available, biotyping strongly suggested a human origin. Four decades later, investigations have found cattle also to be colonized by LA-MRSA, with $88 \%$ of farms positive in Dutch veal-calf rearing units [22]. Only occasional reports exist on dairy farms but in one study in Belgium, up to $15 \%$ of lactating cows in herds with a previous history of MRSA were positive [23]. In general, the occurrence of MRSA in bovine mastitis isolates is well studied and its prevalence seems to be very low [24].

In 2005, a high prevalence of LA-MRSA was found in Dutch pigs in slaughterhouses [25]. This study was undertaken following high colonization rates of pig farmers and relatives without known risk factors for MRSA [13]. Other reports have confirmed these findings in different countries, e.g. Denmark [26], Germany [27], Canada [28] and Belgium [29], and the predominant spa types found were $\mathrm{t} 108, \mathrm{t} 034$, and $\mathrm{t} 011$, all close relatives within CC398. The SCCmec element predominantly found was IV. In 2002 the isolation of this clone from pigs was reported in France for the first time, but the isolate was susceptible to methicillin (MSSA) [30]. In a recent Canadian study [28] an endemic HA-MRSA (US100) strain was found in pigs in addition to CC398. Of the cited porcine studies, the highest percentage of positive farms (living animals, not slaughterhouse) was found in Belgium with 34/50 fattening pig farms studied being positive (68\%). Recent investigations from Asia have demonstrated that pig farms also can act as a reservoir for other sequence types of MRSA, e.g. ST9 in China [31] and Malaysia [32].

In poultry, a Belgian study revealed a new spa type t1456 within CC398 to be present in 2/14 randomly selected broiler farms $(14 \cdot 3 \%)$, but it was not found in 10 layer farms [33]. Similarly, Nemati et al. [34] found $5 / 39$ Belgian broiler farms $(12 \cdot 8 \%)$ to be positive for LA-MRSA (CC398).

A detailed overview on the occurrence in foodproducing animals and derived products is provided elsewhere [5].

To date, clinical infections with LA-MRSA in food-producing animals have been described twice. The first report described a case of post-weaning dermatitis with $20 \%$ mortality in swine from The Netherlands, in which spa type t011 was found [35]. A second report showed LA-MRSA to be present in Belgian bovine clinical and subclinical mastitis isolates [23]. Thus far, the LA-MRSA strains in the living animal have not been reported to possess PVL $[29,36]$. On the contrary, in CC 398 from predominantly healthy persons, a prevalence of $9.4 \%(3 / 32)$ of the PVL toxin was reported [19] although an Asian subclone was suggested for these isolates [37]. Other MRSA clones harbouring PVL have nevertheless been found in living animals [7].

\section{Risk factors for colonization and infection in livestock}

A causal relationship between the use of antibacterial drugs and MRSA has been demonstrated in human medicine for different antimicrobial compounds, e.g. quinolones, glycopeptides and $\beta$-lactams, in a recent meta-analysis [38]. It is probable that similar conditions apply also to animals, particularly as LA-MRSA are often co-resistant to several other antimicrobials as indicated below.

An antimicrobial therapeutic regimen in the strict sense requires a diagnosis. However, many treated animals are not sick at the initiation of the antimicrobial regimen. First, prevention of mainly respiratory and digestive disorders can be done by treating the animals in a known risk period during the production cycle. In addition, healthy animals sharing the same space (barn) with diseased individuals may be treated. Many experts consider preventive therapy necessary in the modern livestock industry. Such practices are common in the majority 
of intensively reared animals like broilers, fattening pigs and veal calves. Of particular concern is that in preventive therapy, deviations from approved posology including underdosing and prolonged duration of treatment are common, and treatment is often initiated without diagnosis [39].

Since most LA-MRSA are resistant to tetracyclines (and trimethoprim) [40], an association between the use of antimicrobials in pig farming [39] and the widespread occurrence of MRSA has been hypothesized [16, 25]. Monitoring of antimicrobial consumption in The Netherlands [41] and Denmark [42] has revealed an increased use of tetracyclines during the last 5 years. So far there has been only one report demonstrating an association between the occurrence of MRSA in livestock and the use of antimicrobials. Van Duijkeren et al. [36] found the number of colonized pigs in farms applying oral group treatments, often with tetracyclines, to be higher compared to farms with no such use of antimicrobials. On the other hand, preliminary results from another ongoing porcine study found no such association [43]. The isolation of LA-MRSA in the case report of dermatitis in swine [35] was preceded by unsuccessful therapy with different antimicrobial classes including cephalosporins (ceftiofur and cefquinome), macrolides, and potentiated sulphonamides. Additional genetic resistance markers, including the multidrug resistance gene $c f r$, could speed up the emergence of MRSA ST398 due to co-selection [44].

$S$. aureus infections in veterinary medicine are seldom treated with $\beta$-lactamase-resistant penicillins. The exception is intramammary use of isoxazolylpenicillins, amoxicillin combined with clavulanic acid and cephalosporins for dairy cows. Despite this practice for several decades, the prevalence of MRSA in bovine $S$. aureus isolates has hitherto been low or absent [45].

A lower prevalence of MRSA was found in sows compared to piglets and finishers in a Belgian survey. In addition this survey revealed a marked difference in the number of MRSA-positive animals between open (94\%) and closed (56\%) farms [29]. This is in line with a Dutch survey which indicated transmission of LA-MRSA within the production chain, e.g. from multiplier to finisher farms [36]. Piglets in multiplier farms can be colonized by different routes or vectors, and longitudinal studies are needed to indicate if the environment, e.g. feed or dust or the sows are the primary source of colonization [25].

\section{Human contact hazard related to livestock}

Epidemiological studies in The Netherlands have indicated that contact with veal calves or pigs was significantly associated with CC398 [13, 46]. In The Netherlands, farmers of swine and veal calves are now considered a defined risk group, and are screened upon admission to hospitals [16].

The occupational hazard for LA-MRSA colonization through (the intensity of) pig contact has been confirmed in Belgian farmers [29], regional German investigations [27] and at an international veterinarian conference in Denmark [47]. Markedly, in two of the studies, current hygiene measures (e.g. wearing a mask) were not found to be protective for MRSA carriage $[29,47]$. These findings were in contrast with conclusions drawn from a Canadian study on horse units [48]. Veterinary practitioners are at risk for MRSA, which is clearly demonstrated in countries with a low overall MRSA prevalence like Denmark [49].

Infections of humans with LA-MRSA have been described since 2004 [13], with an increasing frequency in The Netherlands [46] and Denmark [50]. Examples of severe infections are an aggressive soft tissue infection of a pig-inflicted bite wound [15] and endocarditis [51]. An outbreak in a surgical ward at a Dutch hospital has also been described [16].

\section{Occurrence in horses}

The first report on the isolation of MRSA in horses was published in the 1990s; from 1989 to 1991 MRSA was isolated from 13 mares with metritis in Japan. The source was thought to be a stallion on a stud farm which 10 of the mares had visited. PFGE showed that the isolates had indistinguishable patterns [52, 53]. To date, MRSA has been isolated from horses in Europe, Asia and North America [53-60]. Wound and post-operative infections with MRSA tend to be most common $[1,61]$.

Weese et al. [62] found that $5 \cdot 3 \%$ of horses at a Canadian veterinary teaching hospital and $4.7 \%$ of horses on farms in Canada and the USA [63] were colonized with MRSA. Cuny et al. [12] reported 24 MRSA infections in 768 clinical samples from horses at a veterinary teaching hospital in Austria and an estimated infection rate of 4.8 MRSA cases/1000 equine admissions. In a recent study investigating 110 horses presenting at a Belgian equine clinic $10.9 \%$ of the horses carried MRSA. All isolates in this latter study were LA-MRSA (non-typable by PFGE using 
SmaI digestion) and belonged to spa types t011 and t1451 [59]. In the Netherlands, the percentage of MRSA isolates found in equine clinical samples at the Veterinary Microbiological Diagnostic Center of Utrecht University increased from $0 \%$ in 2002 to $37 \%$ in 2008. MRSA of spa type t064, belonging to MLST ST8 and spa types t011 and t2123, both belonging to the livestock-associated MLST ST398, predominated [64]. In Sweden, one of 300 horses admitted to four equine clinics carried MRSA CC398 spa type t011 [65]. In an equine clinic in Belgium MRSA CC398 spa type t011 were isolated from various infections of 13 hospitalized horses [66]. Busscher et al. [67] did not find any MRSA in 200 healthy horses in the community in The Netherlands, similarly no MRSA was detected in nasal samples of 300 healthy horses from 14 farms in Slovenia by Vengust et al. [68]. MRSA colonization was also not identified in 497 healthy horses on farms in Atlantic Canada [69].

\section{Risk factors for colonization and infection in horses}

Antimicrobial administration within 30 days before admission to a veterinary teaching hospital has been demonstrated to be a risk factor for MRSA colonization in horses [70]. Administration of ceftiofur or aminoglycosides during hospitalization was a risk factor associated with nosocomial MRSA colonization of horses [62].

Weese \& Lefebvre [70] evaluated factors associated with MRSA colonization of horses at the time of admission to a veterinary teaching hospital. Previous colonization of the horse, presence of colonized horses on the farm, admission to the neonatal ICU and admission to a service other than the surgical service were risk factors for community-associated colonization. Weese et al. [62] found that horses colonized at admission at a horse clinic were more likely to develop clinical MRSA infection than those not colonized at admission. The overall incidence rate of nosocomial MRSA colonization was 23/1000 admissions and that of nosocomial MRSA infection was $1 \cdot 8 / 1000$ admissions, with an incidence density of $0 \cdot 88 / 1000$ patient-days. Residence on a farm that housed more than 20 horses was a factor significantly associated with MRSA colonization [63]. In a retrospective multicentre study investigating MRSA infections in 115 horses, previous hospitalization and treatment with gentamicin were associated significantly with CA-MRSA, whereas infected incision sites were associated significantly with HAMRSA [61].

\section{Human contact hazard related to horses}

Occupational or recreational exposure to horses has been incriminated as a risk factor for human MRSA colonization [71]. Nasal carriage was significantly higher in veterinary practitioners $(3.9 \%)$ than in persons not professionally exposed to animals $(0.7 \%)$ or in healthy persons in the Danish community $(<1 \%)$. Exposure to horses was found to be a risk factor [49]. Colonization with MRSA was found in $10 \cdot 1 \%$ of veterinary personnel attending an international equine veterinary conference. An increased risk of being colonized was associated with having treated a horse diagnosed with MRSA or having been personally diagnosed with MRSA in the past year. Handwashing between infected patients and hand-washing between farms was protective [48].

Several studies report that MRSA isolates from horses and people working with horses are indistinguishable and differ from MRSA isolates from humans in the general population [58, 63, 64, 72, 73]. In Austria ST254, spa type t036, SCCmec type IV predominated in horses followed by ST398 (t011, SCCmecIV) and ST1 (t127, SCCmecIV) [72]. In Canada the majority of equine isolates are ST8 (t008, SCCmecIV) classified by PFGE as Canadian MRSA5. The predominance of this clone in horses and horse personnel in Canada suggests that this human-origin clone is horse-adapted [2]. In The Netherlands most equine MRSA are either ST398 spa type t011 or ST8 spa type t064 [64]. From 2000 to 2002, 27 persons were found colonized with MRSA at a Canadian veterinary teaching hospital and 10 horse farms. Only one person, a veterinarian working at the clinic, had clinical infection, and the same strain was isolated from two horses he had cared for [74]. Human skin infections were also reported from three persons working in a foal nursery and MRSA isolates from the humans and the foal were indistinguishable by PFGE [71]. Screening of personnel during an outbreak of MRSA infections of horses at a veterinary teaching hospital showed that persons in close contact with horses were more often MRSA-positive than those without [64].

\section{Occurrence in companion animals}

In 1988 Scott et al. [75] reported the first companion animal-related outbreak of MRSA in a rehabilitation 
geriatric ward where the ward cat was colonized and was implicated as reservoir for re-infection. Infection control measures and removal of the cat led to rapid resolution of the outbreak. Since then the number of reports on infections and colonization with MRSA from companion animals has increased [1]. The transmission of a PVL-positive MRSA strain between humans and a dog has been reported [76]. The available evidence suggests that humans are the source of infection or colonization of companion animals and thus a probable 'humanosis' exists, but animals can act as carriers and pass the infecting strain to humans in contact [77]. Molecular-typing methods support the hypothesis that MRSA of human origin have adapted to companion animals. Clonal complexes tend to be those predominating in people in the region [2].

Healthy animals can be colonized for variable periods of time without developing clinical disease [78], but the overall prevalence of the colonization remains low in dogs and cats [1, 57, 79-83]. Potential differences with the human epidemiology of the disease, particularly the dynamics of colonization in companion animals (e.g. shedding, type of contacts and duration of colonization) may exist and are inadequately identified. A recent study concluded on the lack of transmission of MRSA between apparently healthy dogs in a rescue kennel in the absence of risk factors (e.g. infected MRSA animal) [84].

Little is known about the prevalence of MRSA infections in companion animals. MRSA infections cannot be recognized from their clinical presentations alone because they resemble those caused by methicillin-susceptible S. aureus (MSSA), S. intermedius/pseudintermedius and coagulase-negative staphylococci [85]. S. aureus can give rise to a wide diversity of suppurative infections in animals. In line with that, MRSA have been isolated from diverse skin and STIs including abscesses, dermatitis, postoperative wound infections, and intravenous catheter or surgical implant infections $[1,55,57,73,83,86$, 87-90]. Morris et al. [91] reported that MRSA were significantly more frequently associated with deep pyoderma in dogs than other strains of $S$. aureus. Less frequently MRSA has been isolated from lower urinary tract infection, pneumonia, and chronic rhinitis [55, 83] [92]. A recent study compared MRSA and MSSA infection outcomes in dogs and cats and reported a high survival rate in both groups, probably because of the high frequency of non-invasive infections.

\section{Risk factors for colonization and infection in companion animals}

Little information is available on the risk of antimicrobial usage with regard to MRSA infection or colonization in companion animals. According to case reports quoted above, many animals infected or colonized with MRSA have been treated with antimicrobials prior to the diagnosis. Fluoroquinolone administration has been identified as a risk factor for MRSA infections in dogs and cats [55, 93].

Studies on risk factors other than antimicrobials for MRSA infections in companion animals are scarce. Owners from MRSA-positive households or healthcare workers constitute possible risk factors for this companion animal humanosis $[55,76,93,94]$.

MRSA infections in small animals have also been associated with exposure to medical hospitals, extensive wounds, prolonged hospitalization and immunosuppression [87]. In a retrospective case-control study at three veterinary referral hospitals significant risk factors for the acquisition of a MRSA infection compared to a MSSA infection was the presence of a urinary catheter or a joint infection [93]. Invasive procedures, including the presence of foreign material such as suture material, orthopaedic implants, urinary catheters, central venous lines and chest drains appear to be associated with the persistence of MRSA infections [88, 95]. Other identified risk factors associated with SSIs in general, but highly relevant in particular for staphylococci infections, include: improper surgical site clipping and aseptic preparation before surgery, duration of surgery, duration of anaesthesia, emergency vs. daytime surgery, surgical tissue handling, number of persons present in the operating room, and total length of hospital stay [95]. Small-animal ICUs may be at particular risk for periodic outbreaks of colonization and disease as reported by Weese et al. [96].

Transmission of MRSA infection between dogs apparently can be associated with contamination of the floor in a veterinary clinic (Y. Abbott and F. C. Leonard, personal communication). Thus, in veterinary medicine cleanliness of floors appears to be as important as hand-touch sites in the control of human MRSA infections.

\section{Human contact hazard related to companion animals}

Several studies have examined the prevalence of MRSA in veterinary hospitals in the UK [58, 81, 82]. These studies have shown that veterinary staff and 
their pets have a higher prevalence of MRSA, although they are mostly asymptomatic carriers. A recent study by Moodley et al. [49] showed that MRSA carriage was significantly $(P<0 \cdot 02)$ higher in veterinary practitioners $(3.9 \%)$ than in participants not professionally exposed to animals $(0 \cdot 7 \%)$. The results from this study indicate that veterinary professionals are at risk of MRSA carriage and thus should be informed about this emerging occupational health risk and educated about preventive measures.

MRSA infections in owners having involvement with companion animals such as dogs and cats have been suggested for many years, and evidence for this hazard has increased during recent years [1, 7, 76, 94]. Larger epidemiological studies are required to provide more information on specific risk factors.

\section{CURRENT MANAGEMENT AND THERAPEUTIC OPTIONS}

As discussed above, exposure to antimicrobials is a risk factor for acquisition and spread of MRSA in humans and most probably also in animals. Strategies for prevention and management of MRSA in animals should therefore as far as possible not rely on the use of antimicrobials. Further, such strategies include consideration of the overall use of antimicrobials.

If, for animal welfare reasons, antimicrobial treatment is necessary in individual cases, the risk of emergence of further resistance in the strain of MRSA colonizing the animals needs to be managed, in particular considering zoonotic aspects. Options to manage the risk are, e.g. non-use of antimicrobials that are a last resort for treatment or decolonization of MRSA in humans, contact isolation of the animal during treatment, and monitoring the effects of treatment on resistance in the strain through selective culture and susceptibility tests, should MRSA be reisolated.

\section{LIVESTOCK}

\section{Reduction of antimicrobial selective pressure in livestock}

A reduction of the selective pressure by avoiding routine mass medication, could be a major potential control measure. An additional benefit of this measure would be to preserve the efficacy of the current antimicrobials for veterinary and human use.
To confirm a reduction of antimicrobial consumption and to evaluate a possible benefit, detailed information on the applied therapies is necessary, with respect for animal species and the route of administration. Preferably the indication, the production system (e.g. broiler vs. layer), and the regimens applied needs to be documented in detail (dose, duration, formulation, treatment interval).

\section{Prevention of transmission of MRSA between and within livestock farms}

Given the efficient transmission of LA-MRSA throughout the production chain as shown for swine [36], one way to reduce the dissemination of MRSA from farm to farm would be to improve sanitary control measures between herds and during transport. The piglet suppliers form a first target for this approach, which then might be gradually implemented throughout the food chain. Prevention of trade from MRSA-positive to MRSA-negative herds could be considered.

The stand-alone period is a cornerstone in reducing the persistence of bacterial organisms between production cycles. Research on disinfection measures in different production types are needed in view of the current MRSA situation, primarily in pigs, veal calves and broilers. Conversion of the current stable structures might be considered for a more efficient disinfection via mechanical, physical (drying), chemical (chlorhexidine), and thermal (burning, steaming, cold) cleansing.

\section{Reduce carriers in MRSA-positive livestock farms}

\section{Control options for colonized livestock animals}

Non-antimicrobial management

Sanitary control measures can gradually be implemented in affected livestock, and be conceptually based on other applied eradication programmes in livestock. Given the amount of MRSA-positive animals in many production types, culling of colonized animals may have economical repercussions.

Means of decontaminating colonized animals through, e.g. chlorite bathing could be experimentally studied. LA-MRSA is transmissible between different animal species and between animals, farmers, and other potential sources, and these transmission and re-infection routes need to be documented, including their relative importance for the epidemiology 
of LA-MRSA, to allow preventive measures to be taken.

Strict implementation of hygienic measures might be difficult on livestock farms. In addition there is an apparent inefficiency of current infection control measures including hygiene (biosecurity) on the occurrence of MRSA, especially in pig farms [29, 47]. These observations urgently warrant further specific research.

\section{Antimicrobial treatment in colonized animals}

Decolonization procedures, such as those that are used in individual human patients, in the living animal are logistically difficult to apply to numerous living animals. Such procedures are expensive and because the amount of target animals is high, likely to evoke resistance towards the antimicrobials used.

\section{Control options for infected livestock animals}

Non-antimicrobial management

All other diagnoses need to be excluded prior to defining a bacterial infection. Affected animals need to be immediately separated from healthy animals to prevent further spread. Culling of infected animals is a further option in extreme cases.

Destruction of milk from animals with MRSA mastitis is a prerequisite for avoiding transmission. An option in exceptional cases would be premature drying-off of the infected quarter. The most stringent measure in modern dairy systems is culling of these affected cows. Common procedures to control contagious mastitis need to be followed as recommended in guidelines [97].

Local treatment with antiseptics such as glycerol, chlorhexidine, or povidone iodine might be considered in wound infections. It should be noted that studies on the efficacy of such compounds in MRSAinfected livestock are not available.

The clonal nature of LA-MRSA may offer potential for vaccine development. The efficacy for prevention of colonization also needs to be investigated. Exploration of the efficacy and safety of this and similar measures could be extended to prevention of colonization.

\section{Antimicrobial treatment of infected livestock animals}

Before any antimicrobial treatment of MRSA infections in livestock is considered, the risk for development of further resistance needs to be taken into account. The benefit/risk ratio for antimicrobial treatment needs to be compared with alternatives.

In individual animals where antimicrobials are deemed necessary, the choice of the antimicrobial should always be based on susceptibility testing. Every effort should be made to ensure efficacious treatment by ascertaining an appropriate concentration at the site of the infection.

One report described cases of porcine dermatitis caused by LA-MRSA (CC398), which failed to be cured after empiric therapy with cephalosporins, macrolides and potentiated sulphonamides. Fluoroquinolones were in this case clinically successful [35] but there was no microbiological follow-up.

In different countries, different clones of MRSA are predominant, and antimicrobial susceptibilities may also vary. Most isolates of LA-MRSA are resistant to tetracyclines (including doxycycline) and trimethoprim [34]. During antimicrobial therapy the clinical response needs to be monitored to ensure that further resistance has not developed.

Antimicrobials such as vancomycin, linezolid and teicoplanin are regarded as critically important antimicrobials (CIAs) for the treatment of MRSA infections in human medicine [98]. In veterinary medicine these antimicrobials have no maximum residue limit (MRL), they are therefore not allowed and should not be used in animals intended for food production (Council Regulation EC No. 470/2009). In addition there are ethical concerns about their use in veterinary medicine.

\section{Prevention of transmission of MRSA strains in livestock}

Because of the high colonization frequency in healthy animals and the relatively few documented infections, control options suggested in an earlier section entitled 'Prevention of transmission of MRSA between and within farms' are applicable.

In addition, control options given below in a more specified form for horses and small companion animals [99] have been extrapolated in line with general principles for control of bacterial infections as developed.

- Identification and isolation of animals to minimize the risk of zoonotic transmission.

- Use contact precautions such as protective outerwear, e.g. overalls, aprons or coats and boots or overshoes that are not worn elsewhere. 
- Protective outerwear and all items handled during the treatment of MRSA-positive animals (e.g. boards to drive livestock), should be regarded as potentially contaminated.

- Hand hygiene, e.g. through alcohol gel pouches is essential but must be performed correctly.

- Proper cleaning and disinfection of contaminated environments, including transport vehicles. Special attention should be paid to dust in stables.

- Owners should be informed about the risks and required precautions.

\section{HORSES}

\section{Control options for colonized horses}

\section{Non-antimicrobial management}

A Canadian study describes a case where active screening and strict implementation of infection control protocols resulted in a rapid decrease in number of colonized horses on two farms. At farm A $17 \%$ of the horses and $10 \%$ of the farm personnel were colonized and at farm B $43 \%$ of the horses and $17 \%$ of the farm personnel were colonized with Canadian MRSA-5 which is known to be ST8. The majority of horses eliminated MRSA without antimicrobial treatment. On farm A colonization was eradicated, while only two $(3 \%)$ colonized horses remained on farm B at the end of the study [60]. The authors concluded that the infection control programme was, at least in part, responsible for the decline in colonization (no control group was investigated) and that antimicrobial therapy is not required for eradication of colonization and control of MRSA on horse farms.

\section{Antimicrobial treatment in colonized horses}

Information on the antimicrobial treatment of colonized horses is scarce. Colonization with CMRSA-5 is often transient in adult horses, and colonization can be eliminated if proper measures are taken to prevent re-infection from other horses, people and the environment [100]. However, we do not know if this applies to other MRSA types. Antimicrobial therapy should therefore, if applied, be reserved for persistent colonizations or for those cases where other control measures are impossible.

Applying topical antimicrobials to the nares of horses seems unpractical, although nebulization (e.g. with amikacin) might be an alternative [60]. Safety and efficacy of this therapy needs to be further evaluated before it can be recommended. Oral or parenteral administration could be used, but data on their efficacy are scarce.

\section{Control options for infected horses}

Non-antimicrobial management

A fast and accurate diagnosis is essential for the management of MRSA infections. Therefore, all postoperative infections need to be cultured routinely. In addition, non-healing wounds, and infections not responding to antimicrobial therapy should be suspected. Failure to detect MRSA at an early stage can lead to suboptimal treatment of patients and to late identification of an outbreak, facilitating the spread of MRSA [14]. Local treatment with antiseptics such as glycerol, chlorhexidine, or povidone iodine can be used in wound infections. Studies on the efficacy of such compounds in MRSA-infected horses are not currently available.

\section{Antimicrobial treatment in infected horses}

When considering antimicrobial therapy of animals infected with MRSA, the risk for further development of resistance in the infecting strain needs to be considered. The choice of the antimicrobial should always be based on susceptibility testing. Many equine MRSA are still susceptible to commonly used 'routine' antimicrobials. In different countries different clones of MRSA are predominant and also antimicrobial susceptibilities may vary between isolates. During antimicrobial therapy the clinical response should be monitored carefully. Studies comparing the efficacy of different antimicrobial strategies in infected horses are lacking, but are urgently needed.

The efficacy and safety of antimicrobials that are critical for MRSA treatment in human medicine, e.g. vancomycin, linezolid and teicoplanin, have not been assessed in horses and similarly to livestock there are ethical concerns about using them in veterinary medicine. Therefore the use of these antimicrobials is not advisable.

\section{Prevention of transmission of MRSA strains between horses}

Guidelines on the management of MRSA in veterinary practices have been developed, e.g. by the British Small Animal Veterinary Association (BSAVA) [99] and are applicable also to equine hospitals. It is 
recommended that patients diagnosed with or suspected of MRSA infections are isolated in order to minimize the risk of nosocomial and zoonotic transmission. MRSA-infected animals should be nursed using barrier nursing precautions and staff contact should be limited to what is essential. Infected horses should only be handled using contact precautions such as protective outerwear, e.g. overalls, aprons or coats and boots or overshoes that are not worn elsewhere, gloves and masks. MRSA-infected wounds should be covered with clean bandages if possible. Personnel must avoid contaminating themselves. All items handled during the treatment of a MRSApositive patient should be regarded as potentially contaminated, e.g. electric shavers.

Transmission of the organism on the hands is thought to be an important route of transmission within human and veterinary hospital settings. Therefore, hand hygiene should be an essential part of any infection control programme. Hand-washing should be carried out between patients. Alcohol gel pouches for wearing on uniforms can be used as a rapid and convenient method of hand sanitizing [1].

Proper cleaning and disinfection of contaminated environments is also highly recommended, because widespread contamination of the environment of a veterinary hospital environment has been reported $[14,64,100]$. Environmental transmission may be of greater importance in stables than in hospitals [1] as horse stables are often very dusty.

Routine screening of all horses before admission in order to identify colonized or infected animals could help to prevent the spread of MRSA, but is costly. Screening of hospitalized horses which have been in contact with MRSA-positive horses or personnel is recommended.

Owners of infected horses need to be informed about the risks of MRSA and the precautions they should take. Furthermore, the recommendation from BSAVA that owners visiting their infected animal should not visit other patients at the clinic [99] is also applicable to horse practices.

\section{COMPANION ANIMALS}

Given the available evidence that MRSA found in companion animals share the same genetic background as MRSA strains common in human infections, experience gained from MRSA-control in human medicine is likely to be applicable to companion animals.

\section{Control options for colonized companion animals}

\section{Non-antimicrobial management}

Routine decolonization therapy is not recommended in humans or animals that have mucosae colonized with MRSA [101]. At present there is no evidence of the effectiveness of various procedures to decolonize companion animals. Non-antimicrobial management may include baths with, e.g. chlorhexidine which may help to decontaminate the coat but does not address other colonized sites such as oropharynx.

Persistent colonization has not yet been reported in companion animals, and some pets appear to eliminate MRSA carriage spontaneously if recolonization is prevented [83]. In one recent study, MRSA carriage in all healthy carrier dogs was transient and resolved without antimicrobial decolonization therapy in the regularly cleaned and disinfected environment [84]. This may constitute an important factor for MRSA control but long-term studies are needed to confirm this, particularly within a home environment.

\section{Antimicrobial treatment of colonized companion animals}

Given the potential for MRSA selection including additional resistance markers, antimicrobial therapy could be questioned except for cases with persistent colonizations or for those cases where other control measures are impossible. Antimicrobial therapy may be considered in individual MRSA colonized animals as an option to control transmission of MRSA between animals or from animals to humans. In addition, strategic control programmes could include decolonization in specific circumstances.

As yet, no antimicrobials for veterinary medicine have been adequately studied and approved for local or systemic application intended to decolonize MRSA carrier animals.

Case reports of successful eradication therapy in one MRSA colonized dog with a combination of ciprofloxacin and rifampin and another with rifampin and doxycycline have been described [75, 76, 94]. Decolonization with antimicrobials may involve a risk of selection of MRSA strains resistant to the agent applied. Of note is a report on high-level mupirocin resistance $(>256 \mathrm{mg} / \mathrm{l})$ of $S$. aureus isolated in two cases of post-operative infections in dogs and in one case of lower urinary tract infection in a cat [83]. Fusidic acid resistance was also reported in an MRSA isolate from a seal [58]. 
Topical agents used in humans for decolonization are mupirocin and fusidic acid alone or in combination with other topical agents such as chlorhexidine or bacitracin. Systemic antimicrobials used include cotrimoxazole (trimethoprim + sulfonamides), rifampicin, and doxycycline, in general given by the oral route [102, 103].

As for livestock and horses, the use of antimicrobials in pets that are critical for MRSA treatment in humans is controversial, due to the risk for development of resistance against those agents. In some countries veterinary use of some antimicrobials, including mupirocin is limited to exceptional conditions or prohibited by law.

\section{Control options for infected companion animals}

\section{Non-antimicrobial management}

In some cases of wound infections in systemically healthy animals, meticulous local wound management may avoid the need for local or parenteral antimicrobial therapy [85]. This was the case described in a report on a $\operatorname{dog}$ with a wound MRSA infection that had been treated with marbofloxacin and resolved after stopping antimicrobial therapy, debridement of the wound and administration of anti-inflammatory steroids [104]. Superficial infections such as uncomplicated wound or incision infections can be treated with a variety of topical agents, including silver sulfadiazine, or a combination of $1 \%$ silver sulfadiazine and $0 \cdot 2 \%$ chlorhexidine digluconate [105]. Whenever topical therapy alone is used, close monitoring progression of local disease or development of bacteraemia and systemic disease is required [105].

\section{Antimicrobial treatment of infected companion animals}

As the clinical manifestations of MRSA infections in animals are variable, no single treatment protocol is suitable for all animals. The treatment must be tailored to the individual patient [85]. When choosing a treatment plan, the risk for development of resistance in the MRSA-infecting strain needs to be considered. In addition, several factors should be taken into account: (i) the susceptibility profile of the MRSA isolated from the animal patient, (ii) the severity of the infection and presence of systemic disease (fever, leukocytosis), (iii) the patient's underlying disease or any comorbidity [85]. Local antimicrobial therapy may be an option in certain cases [89], while in some patients systemic antimicrobial therapy may be required. The decision of treatment or eventual animal euthanasia should take into account available national veterinary guidelines for infection control. Information on treatment and outcome of MRSA infections in companion animals is scarce.

Tomlin and colleagues [90] described treatment of 11 MRSA infections in dogs: six occurred after surgical treatment, two as complication of wounds, and three in dogs with recurrent pyoderma secondary to primary diseases such as atopic dermatitis, demodicosis and hypothyroidism. Wound and surgical treatment as well as oral antibiotic treatment (trimethoprimsulfadiazine, ciprofloxacin, enrofloxacin and clindamycin) based on culture and susceptibility testing clinically cured infection in 9/11 dogs. Clindamycin could be a potential candidate for MRSA skin, soft tissue and bone infections. However, Rich et al. [106] identified inducible resistance to clindamycin which could compromise success of therapy.

MRSA sepsis is rare in companion animals. Antimicrobial therapy of these patients is challenging as some MRSA strains may not be susceptible to any of bactericidal agents which can be administered intravenously (e.g. cephalosporins, aminoglycosides, fluoroquinolones) which would generally be chosen. Treatment options for human invasive MRSA infections currently include vancomycin, linezolid, daptomycin, tigecycline, and quinupristin/dalfopristin but adequate data to support the use of these compounds in companion animals are not available. Resistance to new antimicrobials (linezolid, daptomycin, tigecycline) has also been reported [107]. Additionally, a number of compounds for human use to combat the growing resistance problems are in development, including novel glycopeptides (dalbavancin, telavancin, oritavancin), and next-generation cephalosporins (ceftobiprole, ceftaroline), which demonstrate excellent in vitro activity against MRSA, vancomycinintermediate $S$. aureus (VISA), and vancomycinresistant $S$. aureus (VRSA).

Veterinary use of antimicrobials that are regarded as CIAs for human MRSA infections (e.g glycopeptides, oxazolidones, tigecycline, streptogramins) is undesirable as it will increase the risk for emergence and spread of resistance.

\section{Prevention of transmission of MRSA between companion animals}

Guidelines for control and prevention of MRSA infection in small animals have been prepared, e.g. by 
BSAVA [99]. Stringent household infection control practices, in particular frequent hand hygiene and avoiding high-risk contact are important factors to minimize the risk of becoming colonized. In the event of rare situations where MRSA infections are uncontrolled in people in the household and the entire family is undergoing eradication therapy, kennelling the pet, preferably using contact isolation to other pets, for weeks is a reasonable option. This may allow the clearance of colonization and avoid crosscontamination. Little is known about the nature and length of colonization in companion animals. Further studies are required to reach conclusions.

Use of latex gloves and protection measures such as masks and eye protection may be helpful provided they are appropriately used and changed between patients. For instance, it is recommended to rub the hands with alcohol immediately after the gloves are removed. Hand hygiene and disinfection of surfaces and equipment should be carried out between all patients. Finally, additional barrier precautions must be considered based on the conditions present in the hospital.

Additional simple strategies can effectively reduce the risk of nosocomial infections-including MRSA - in small animal patients. First, limitation of the extrinsic risk factors is the most logical approach: (i) invasive procedures should be restricted, (ii) surgical interventions should be designed to avoid the many risk factors for surgical site infections, (iii) indwelling urinary catheters should be removed as soon as the patient's condition allows it, (iv) the consequences of antibiotic use in dogs and cats with indwelling urinary catheters should also be evaluated, and (v) proper care of intravenous and urinary catheters is essential to prevent complications [95]. Protocols for asepsis prior to and during placement of the catheter should exist. ICUs may be at particular risk for periodic outbreaks of colonization and disease, but can be curtailed by barrier precautions, and hand hygiene [96].

In veterinary hospitals, animals with suspected MRSA infections (animals with non-healing wounds, with non-antibiotic responsive infections or with nosocomial infections), animals from known MRSApositive households, or those belonging to healthcare workers should be screened for MRSA colonization. The implementation of the 'Search-and-destroy' strategy as successfully applied in human medicine by certain Northern European countries could possibly be applied in small animal hospitals, given that the time for MRSA detection and the length of hospital stay allows for implementation of additional control options. Concerns have been raised about dogs involved in hospital visitation programmes such as therapy pets [108].

\section{PERSONS IN CONTACT WITH LIVE ANIMALS}

There is a need for educational programmes for veterinarians to be organized by the competent authorities taking into account the regional differences in occurrence of MRSA in the hospital, community and animal husbandry. Such programmes should include information for farmers and owners of infected animals.

Cooperation between the medical and veterinary professions is a vital requirement not only to identify human carriers but also to implement effective control measures. Owners, farmers and their household contacts (possibly including children, elderly, and immunosuppressed people) are at risk after exposure to an infected/colonized MRSA animal. Basic hygienic measures have been shown to be protective in practice [48], although knowledge and compliance with protective behaviour that helps to reduce the risk of zoonotic transmission in general could substantially be improved among veterinarians [48, 109]. Clearly, for all people having contact with the living animal, appropriate hygiene and covering wounds and skin lesions are the two cornerstones in minimizing the spread of MRSA between individuals, including the transmission from animals to humans and vice versa. Essential are correct hand-washing, alcohol-based hand sanitizers and their availability, e.g. in consulting rooms, animal units, and on farms. The use of disposable aprons, gloves, masks, and eye protection are appropriate especially for contact with suspected wounds, body fluids or other contaminated materials.

Surveillance of veterinary staff, farmers or owners for MRSA carriage is controversial and it is an issue that requires confidentiality. Ethical and privacy concerns needs to be considered during the preparation of further guidelines.

\section{SUMMARY ASSESSMENT}

\section{Conclusions on ecology and epidemiology}

General

- MRSA is resistant to virtually all $\beta$-lactam agents. In human medicine there is evidence that the use 
of a variety of antimicrobials is a major risk factor for colonization and infection.

- While in companion animals the MRSA strains are evolutionarily related to different typical humanassociated MRSA clones, this is not the case for the clonally spread MRSA CC398 found in foodproducing animals.

- All the major lineages of MRSA strains in companion animals, horses and in livestock are able to infect animals and humans. Severe infections have been described.

\section{Livestock}

- The recently discovered MRSA strain, CC398, has emerged in the livestock production chain preharvest, mainly in intensified production systems, e.g. fattening pigs, veal calves and broilers.

- Both efficient transmission of CC398 between farms and high within-herd prevalence have been documented in many countries worldwide.

- As with human medicine, antimicrobial consumption must be considered a driving force in the emergence and spread of CC398, and additional studies confirming this hypothesis are urgently needed.

- Molecular studies support the hypothesis that coselection by non- $\beta$-lactam agents probably contributes to the high prevalence of CC398.

- Monitoring of antimicrobial consumption in veterinary medicine is lacking in most countries. Such monitoring is required to provide additional evidence for the causal relationship with the occurrence of MRSA CC398.

\section{Horses}

- Horses can be colonized and infected by specific lineages of MRSA, of which CC398 is one.

- Case studies suggest equine hospitals to be at high risk, and post-surgical infections can lead to epidemics within such settings.

- Studies indicate that antimicrobial use is a risk factor for MRSA carriage in horses, which agrees with the evidence from MRSA epidemiology in human medicine.

\section{Companion animals}

- Intensive contact of humans with companion animals results in the exchange of typically human MRSA clones.
- MRSA contamination can lead to colonization, to infection especially after surgery, and to crosstransmission between owners, veterinary personnel and other companion animals.

- Studies indicate that antimicrobial use is a risk factor for MRSA carriage in companion animals, which agrees with the evidence from MRSA epidemiology in human medicine.

\section{Conclusion on control and therapeutic options}

\section{General}

- Based upon extrapolations from human medicine, biosecurity and reduction of antimicrobial selection pressure are cornerstones in constraining the spread of MRSA in animal husbandry.

- Biosecurity and infection control measures depend on the animal species and specific settings.

- Hygiene measures such as hand disinfection and adequate wound management are essential.

- For surveillance purposes, records for antimicrobial consumption need to be detailed, including, e.g. information of the animal species and regimen applied (e.g. dose and route of administration), in order to evaluate the compliance with and effect of antibiotic policies.

- Limitation of veterinary use of critical and new agents for MRSA infections in humans needs to be considered. No MRLs are set for such substances and they cannot be used for food-producing animals. Their use in companion animals and nonfood-producing horses is also questionable, due to the risk for development of resistance against these agents and subsequent spread of resistant bacteria to humans.

- Studies need to document the long-term carriage of MRSA, and find efficient ways to decolonize animals and to clear different animal husbandry settings.

- Blind, randomized controlled trials as well as a pro-active surveillance of MRSA in different animal species is required to follow-up evolution and to provide evidence with regard to efficient and affordable control strategies.

\section{Livestock}

- The extensive use of antimicrobials for prevention of disease appears to be an important risk factor for the spread of MRSA although data are still sparse. 
- Taking into account the occurrence of CC398 in different member states the multi-faceted approach is advisable whereby infection control strategies and surveillance are integrated.

- Biosecurity measures that disrupt the spread within and between farms need to be documented and investigated for their efficacy and long-term effect. A focus on avoiding transmission via trade is advisable.

\section{Horses and companion animals}

- Well controlled hygiene and quarantine measures are needed to clear hospital epidemics.

- Strategies that effectively reduce the risk of hospital-acquired infections, including MRSA, need to be applied. One component of such strategies would be to limit the prophylactic use of antimicrobials related to surgery.

Persons in contact with live animals

- Close collaboration between human and veterinary experts, coupled with appropriate education is necessary to develop adequate management guidelines.

- Risk mitigation measures to limit the spread of MRSA between animals needs to consider humans in contact with animals.

\section{ACKNOWLEDGEMENTS}

The authors sadly report that one of the co-authors, Professor Fritz Ungemach, passed away on 20 December 2009. The information contained in this publication does not necessarily reflect the opinion or the position of the European Medicines Agency.

\section{DECLARATION OF INTEREST}

None.

\section{REFERENCES}

1. Leonard FC, Markey BK. Meticillin-resistant Staphylococcus aureus in animals: a review. Veterinary Journal 2008; 175: 27-36.

2. Weese JS, Van Duijkeren E. Methicillin-resistant Staphylococcus aureus and Staphylococcus pseudintermedius in veterinary medicine. Veterinary Microbiology. doi:10.1016/j.vetmic.2009.01.039.

3. Safdar N, Bradley EA. The risk of infection after nasal colonization with Staphylococcus aureus. American Journal of Medicine 2008; 121 : 310-315.
4. Hermans K, et al. MRSA clone ST398-SCCmecIV as a cause of infections in an equine clinic. Vlaams Diergeneeskundig Tijdschrift 2008; 77: 429-433.

5. EFSA. 2009 European Food Safety Authority. Assessment of the Public Health significance of meticillin resistant Staphylococcus aureus (MRSA) in animals and foods Scientific Opinion of the Panel on Biological Hazards.

6. de Lencastre H, Oliveira D, Tomasz A. Antibiotic resistant Staphylococcus aureus: a paradigm of adaptive power. Current Opinion in Microbiology 2007; 10: 428 435.

7. Morgan M. Methicillin-resistant Staphylococcus aureus and animals: zoonosis or humanosis? Journal of Antimicrobial Chemotherapy 2008; 62: 1181-1187.

8. Haesebrouck FVD, et al. MRSA from animals: a public treat? Vlaams Diergeneeskundig Tijdschrift 2006; 75: 254-261.

9. Salgado CD, Farr BM, Calfee DP. Communityacquired methicillin-resistant Staphylococcus aureus: a meta-analysis of prevalence and risk factors. Clinical Infectious Diseases 2003; 36: 131-139.

10. Bartels MD, et al. Rapid increase of genetically diverse methicillin-resistant Staphylococcus aureus, Copenhagen, Denmark. Emerging Infectious Diseases 2007; 13: 1533-1540.

11. Anon. Prudent use in animals, chapter 3. London, UK, House of Lords. Science and Technology - Seventh Report. [Resistance to antibiotics and other antimicrobial agents 3.14.1998], 1998.

12. Cuny C, et al. Emergence of MRSA infections in horses in a veterinary hospital: strain characterization and comparison with MRSA from humans. Eurosurveillance 2006 ; 11: 44-47.

13. Voss A, et al. Methicillin-resistant Staphylococcus aureus in pig farming. Emerging Infectious Diseases 2005; 11: 1965-1966.

14. Weese JS. A review of multidrug resistant surgical site infections. Veterinary and Comparative Orthopaedics and Traumatology 2008; 21 : 1-7.

15. Declercq $\mathbf{P}$, et al. Complicated community-acquired soft tissue infection by MRSA from porcine origin. Infection 2008; 36: 590-592.

16. Wulf MW, et al. First outbreak of methicillin-resistant Staphylococcus aureus ST398 in a Dutch hospital, June 2007. Eurosurveillance 2008; 13.

17. Appelbaum PC. Microbiology of antibiotic resistance in Staphylococcus aureus. Clinical Infectious Diseases 2007; 45 (Suppl.): S165-S170.

18. David MZ, et al. What is community-associated methicillin-resistant Staphylococcus aureus? Journal of Infectious Diseases 2008; 197: 1235-12343.

19. van Loo I, et al. Emergence of methicillinresistant Staphylococcus aureus of animal origin in humans. Emerging Infectious Diseases 2007; 13: 1834 1839.

20. Rasschaert G, et al. Comparison of fingerprinting methods for typing methicillin-resistant Staphylococcus aureus sequence type 398. Journal of Clinical Microbiology 2009; 47: 3313-3322. 
21. Devriese LA, Van Damme LR, Fameree L. Methicillin (cloxacillin)-resistant Staphylococcus aureus strains isolated from bovine mastitis cases. Zentralblatt fur Veterinarmedizin B 1972; 19: 598-605.

22. Graveland H, et al. Methicillin-resistant Staphylococcus aureus (MRSA) in veal calf farmers and veal calves in The Netherlands. ASM Conference on: Antimicrobial Resistance in Zoonotic Bacteria and Foodborne Pathogens, Copenhagen, Denmark, 15-18 June 2008, B84.

23. Vanderhaeghen W, et al. Methicillin-resistant Staphylococcus aureus (MRSA) associated with clinical and subclinical mastitis in Belgian cows. Veterinary and Agrochemical Research Centre, Brussels, Belgium, Scientific Report 2007-2008, 2008, pp. 30-34.

24. Hendriksen RS, et al. Prevalence of antimicrobial resistance among bacterial pathogens isolated from cattle in different European countries: 2002-2004. Acta Veterinaria Scandinavica 2008; 50: 28.

25. De Neeling aJ, et al. High prevalence of methicillin resistant Staphylococcus aureus in pigs. Veterinary Microbiology 2007; 122: 366-372.

26. Guardabassi L, Stegger M, Skov R. Retrospective detection of methicillin resistant and susceptible Staphylococcus aureus ST398 in Danish slaughter pigs. Veterinary Microbiology 2007; 122: 384-386.

27. Meemken D, et al. Occurrence of MRSA in pigs and in humans involved in pig production-preliminary results of a study in the northwest of Germany [in German]. Deutsche Tierarztliche Wochenschrifte 2008; 115: 132-139.

28. Khanna T, et al. Methicillin resistant Staphylococcus aureus colonization in pigs and pig farmers. Veterinary Microbiology 2008; 128: 298-303.

29. Denis O, et al. Methicillin-resistant Staphylococcus aureus ST398 in swine farm personnel, Belgium. Emerging Infectious Diseases 2009; 15: 1098-1101.

30. Armand-Lefevre L, Ruimy R, Andremont A. Clonal comparison of Staphylococcus aureus isolates from healthy pig farmers, human controls, and pigs. Emerging Infectious Diseases 2005; 11: 711-714.

31. Wagenaar JA, et al. Unexpected sequence types in livestock associated methicillin-resistant Staphylococcus aureus (MRSA): MRSA ST9 and a single locus variant of ST9 in pig farming in China. Veterinary Microbiology 2009; 139: 405-409.

32. Neela V, et al. Prevalence of ST9 MRSA among pigs and pig handlers in Malaysia. Journal Clinical Microbiology 2009; 47: 4138-4140.

33. Persoons D, et al. Methicillin-resistant Staphylococcus aureus in poultry. Emerging Infectious Diseases 2009; 15: 452-453.

34. Nemati M, et al. Antimicrobial resistance of old and recent Staphylococcus aureus isolates from poultry: first detection of livestock-associated methicillinresistant strain ST398. Antimicrobial Agents and Chemotherapy 2008; 52: 3817-3819.

35. van Duijkeren E, et al. Methicillin-resistant Staphylococcus aureus in pigs with exudative epidermitis. Emerging Infectious Diseases 2007; 13: 1408-1410.
36. van Duijkeren E, et al. Transmission of methicillinresistant Staphylococcus aureus strains between different kinds of pig farms. Veterinary Microbiology 2008; 126: 383-389.

37. Yu F, et al. Prevalence of Staphylococcus aureus carrying Panton-Valentine leukocidin genes among isolates from hospitalised patients in China. Clinical Microbiology and Infection 2008; 14: 381-384.

38. Tacconelli E, et al. Does antibiotic exposure increase the risk of methicillin-resistant Staphylococcus aureus (MRSA) isolation? A systematic review and metaanalysis. Journal of Antimicrobial Chemotherapy 2008; 61: 26-38.

39. Timmerman T, et al. Quantification and evaluation of antimicrobial drug use in group treatments for fattening pigs in Belgium. Preventive Veterinary Medicine 2006; 74: 251-263.

40. Kadlec K, Schwarz S. Identification of a novel trimethoprim resistance gene, dfrK, in a methicillinresistant Staphylococcus aureus ST398 strain and its physical linkage to the tetracycline resistance gene tet(L). Antimicrobial Agents and Chemotherapy 2009; 53: 776-778.

41. MARAN. Monitoring of antimicrobial resistance and antibiotic usage in animals in the Netherlands in 2005 (http://www.cvi.wur.nl/NR/rdonlyres/A906A4C0-A458423E-B932-28F222385988/83791/MARAN_2007_def3. pdf), 2005. Accessed 26 November 2009.

42. DANMAP. The Danish integrated antimicrobial resistance monitoring and research programme 2006 (http://www.food.dtu.dk/Default.aspx?ID=9604). Accessed 26 November 2009.

43. Broens EM, et al. Prevalence study and risk factor analysis of NT-MRSA in pigs in the Netherlands. ASM Conference on Antimicrobial Resistance in Zoonotic Bacteria and Foodborne Pathogens, Copenhagen, Denmark, 15-18 June 2009, A19.

44. Kehrenberg C, et al. Methicillin-resistant and -susceptible Staphylococcus aureus strains of clonal lineages ST398 and ST9 from swine carry the multidrug resistance gene cfr. Antimicrobial Agents and Chemotherapy 2009; 53: 779-781.

45. Virgin JE, et al. Short communication: methicillinresistant Staphylococcus aureus detection in US bulk tank milk. Journal of Dairy Science 2009; 92: 49884991.

46. Van Rijen MM, Van Keulen PH, Kluytmans JA. Increase in a Dutch hospital of methicillin-resistant Staphylococcus aureus related to animal farming. Clinical Infectious Diseases 2008; 46: 261-263.

47. Wulf MW, et al. Prevalence of methicillin-resistant Staphylococcus aureus among veterinarians: an international study. Clinical Microbiology and Infection 2008; 47: 29-34.

48. Anderson ME, Lefebvre SL, Weese JS. Evaluation of prevalence and risk factors for methicillin-resistant Staphylococcus aureus colonization in veterinary personnel attending an international equine veterinary conference. Veterinary Microbiology 2008; 129: 410417. 
49. Moodley A, et al. High risk for nasal carriage of methicillin-resistant Staphylococcus aureus among Danish veterinary practitioners. Scandinavian Journal of Work, Environment \& Health 2008; 34: 151-157.

50. Lewis HC, et al. Pigs as source of methicillin-resistant Staphylococcus aureus CC398 infections in humans, Denmark. Emerging Infectious Diseases 2008; 14: 1383-1389.

51. Ekkelenkamp MB, et al. Endocarditis due to meticillin-resistant Staphylococcus aureus originating from pigs [in Dutch]. Nederlands tijdschrift voor geneeskunde 2006; 150: 2442-2447.

52. Anzai T, et al. Isolation of methicillin-resistant Staphylococcus aureus (MRSA) from mares with metritis and its zooepidemiology. Journal of Equine Science 1996; 7: 7-11.

53. Shimizu A, et al. Genetic analysis of equine methicillinresistant Staphylococcus aureus by pulsed-field gel electrophoresis. Journal of Veterinary Medical Science 1997; 59: 935-937.

54. Witte W, et al. Methicillin-resistant Staphylococcus aureus ST398 in humans and animals, Central Europe. Emerging Infectious Diseases 2007; 13: 255258.

55. Baptiste KE, et al. Methicillin-resistant staphylococci in companion animals. Emerging Infectious Diseases 2005; 11: 1942-1944.

56. Hartmann FA, Trostle SS, Klohnen AA. Isolation of methicillin-resistant Staphylococcus aureus from a postoperative wound infection in a horse. Journal of the American Veterinary Medical Association 1997; 211: 590-592.

57. Middleton JR, et al. Surveillance of Staphylococcus aureus in veterinary teaching hospitals. Journal of Clinical Microbiology 2005; 43: 2916-2919.

58. O'Mahony R, et al. Methicillin-resistant Staphylococcus aureus (MRSA) isolated from animals and veterinary personnel in Ireland. Veterinary Microbiology 2005; 109: 285-296.

59. van Den Eede A, et al. High occurrence of methicillinresistant Staphylococcus aureus ST398 in equine nasal samples. Veterinary Microbiology 2009; 133: 138144.

60. Weese JS, Rousseau J. Attempted eradication of methicillin-resistant Staphylococcus aureus colonisation in horses on two farms. Equine Veterinary Journal $2005 ; 37$ : 510-514.

61. Anderson ME, et al. Retrospective multicentre study of methicillin-resistant Staphylococcus aureus infections in 115 horses. Equine Veterinary Journal 2009; 41: 401-405.

62. Weese JS, et al. Methicillin-resistant Staphylococcus aureus in horses at a veterinary teaching hospital: frequency, characterization, and association with clinical disease. Journal of Veterinary Internal Medicine 2006; 20: 182-186.

63. Weese JS, et al. Community-associated methicillinresistant Staphylococcus aureus in horses and humans who work with horses. Journal of the American Veterinary Medical Association 2005; 226: 580-583.
64. Van Duijkeren E, et al. Methicillin-resistant Staphylococcus aureus in horses and horse personnel: an investigation of several outbreaks. Veterinary Microbiology. doi:10.1016/j.vetmic.2009.08.009.

65. SVARM. 2007: Swedish veterinary antimicrobial resistance monitoring 2007. (http://www.sva.se/upload/ pdf $/ \mathrm{Tj} \% \mathrm{C} 3 \%$ A4nster $\%$ 20och $\% 20$ produkter/Tryck saker/SVARM_2007\%5B1\%5D.pdf). Accessed 21 November 2009.

66. Hermans KLU, et al. MRSA clone ST398-SCCmecIV as a cause of infections in an equine clinic. Vlaams Diergeneeskundig Tijdschrift 2008; 77: 429-433.

67. Busscher JF, van Duijkeren E, Sloet van Oldruitenborgh-Oosterbaan MM. The prevalence of methicillin-resistant staphylococci in healthy horses in the Netherlands. Veterinary Microbiology 2006; 113: 131-136.

68. Vengust M, et al. Methicillin-resistant staphylococcal colonization in clinically normal dogs and horses in the community. Letters in Applied Microbiology 2006; 43 : 602-606.

69. Burton S, et al. Staphylococcus aureus colonization in healthy horses in Atlantic Canada. Canadian Veterinary Journal 2008; 49: 797-799.

70. Weese JS, Lefebvre SL. Risk factors for methicillinresistant Staphylococcus aureus colonization in horses admitted to a veterinary teaching hospital. Canadian Veterinary Journal 2007; 48: 921-926.

71. Weese JS, et al. An outbreak of methicillin-resistant Staphylococcus aureus skin infections resulting from horse to human transmission in a veterinary hospital. Veterinary Microbiology 2006; 114: 160-164.

72. Cuny C, et al. Clusters of infections in horses with MRSA ST1, ST254, and ST398 in a veterinary hospital. Microbial Drug Resistance 2008; 14: $307-$ 310.

73. Seguin JC, et al. Methicillin-resistant Staphylococcus aureus outbreak in a veterinary teaching hospital: potential human-to-animal transmission. Journal of Clinical Microbiology 1999; 37: 1459-1463.

74. Weese JS. Methicillin-resistant Staphylococcus aureus in horses and horse personnel. Veterinary Clinics of North America: Equine Practice 2004; 20 : 601-613.

75. Scott GM, et al. Cross-infection between animals and man: possible feline transmission of Staphylococcus aureus infection in humans? Journal of Hospital Infection $1988 ; 12$ : 29-34.

76. van Duijkeren E, et al. Human-to-dog transmission of methicillin-resistant Staphylococcus aureus. Emerging Infectious Diseases 2004; 10: 2235-2237.

77. Strommenger B, et al. Molecular characterization of methicillin-resistant Staphylococcus aureus strains from pet animals and their relationship to human isolates. Journal of Antimicrobial Chemotherapy 2006; 57 : 461465.

78. Weese JS. Emergence of methicillin-resistant Staphylococcus aureus in pets: implications for animal and human health. In: Proceedings of the American College of Internal Veterinary Medicine (http:// www.vin.com/Members/Proceedings/Proceedings.plx? 
$\mathrm{CID}=$ acvim2005\&PID $=$ pr09532\&O=VIN), 2005. Accessed 10 January 2010.

79. Murphy C, et al. Occurrence of antimicrobial resistant bacteria in healthy dogs and cats presented to private veterinary hospitals in southern Ontario: a preliminary study. Canadian Veterinary Journal 2009; 50: 1047-1053.

80. Abraham JL, et al. Surveillance of healthy cats and cats with inflammatory skin disease for colonization of the skin by methicillin-resistant coagulasepositive staphylococci and Staphylococcus schleiferi ssp. schleiferi. Veterinary Dermatology 2007; 18: 252259.

81. Hanselman BA, Kruth S, Weese JS. Methicillinresistant staphylococcal colonization in dogs entering a veterinary teaching hospital. Veterinary Microbiology 2008; 126: 277-281.

82. Loeffler A, et al. Prevalence of methicillin-resistant Staphylococcus aureus among staff and pets in a small animal referral hospital in the UK. Journal of Antimicrobial Chemotherapy 2005; 56: 692-697.

83. Weese JS, et al. Suspected transmission of methicillinresistant Staphylococcus aureus between domestic pets and humans in veterinary clinics and in the household. Veterinary Microbiology 2006; 115: 148155.

84. Loeffler A, et al. Lack of transmission of methicillinresistant Staphylococcus aureus (MRSA) between apparently healthy dogs in a rescue kennel. Veterinary Microbiology. doi:10.1016/j.vetmic.2009.08.001.

85. Lloyd DH, Boag AK, Loeffler A. Dealing with MRSA in companion animals practice. European Journal of Companion Animal Practice 2007; 17: 85-93.

86. Bender JB, et al. Isolation of methicillin-resistant Staphylococcus aureus from a non-healing abscess in a cat. Veterinary Record 2005; 157: 388-389.

87. Duquette RA, Nuttall TJ. Methicillin-resistant Staphylococcus aureus in dogs and cats: an emerging problem? Journal of Small Animal Practice 2004; 45: 591-597.

88. Leonard F, et al. Methicillin-resistant Staphylococcus aureus isolated from a veterinary surgeon and five dogs in one practice. Veterinary Record 2006; 158: 155-159.

89. Owen MR, Moores AP, Coe RJ. Management of MRSA septic arthritis in a dog using a gentamicinimpregnated collagen sponge. Journal of Small Animal Practice 2004; 45: 609-612.

90. Tomlin J, et al. Methicillin-resistant Staphylococcus aureus infections in 11 dogs. Veterinary Record 1999; 144: 60-64.

91. Morris DO, et al. Screening of Staphylococcus aureus, Staphylococcus intermedius, and Staphylococcus schleiferi isolates obtained from small companion animals for antimicrobial resistance: a retrospective review of 749 isolates (2003-04). Veterinary Dermatology 2006; 17: 332-337.

92. Gortel K, et al. Methicillin resistance among staphylococci isolated from dogs. American Journal of Veterinary Research 1999; 60: 1526-1530.
93. Faires M, Weese S. Risk factors associated with methicillin resistant Staphylococcus aureus infections in dogs and cats. ASM Conference on Antimicrobial Resistance in Foodborne Pathogens and Zoonotic Bacteria, Copenhagen, Denmark, 15-18 June 2008, S8:3.

94. van Duijkeren E, et al. Transmission of a PantonValentine leucocidin-positive, methicillin-resistant Staphylococcus aureus strain between humans and a dog. Journal of Clinical Microbiology 2005; 43: 62096211.

95. Gaschen. Nosocomial infection: prevention and approach, 2008 (http://www.vin.com/proceedings/ Proceedings.plx?CID $=$ WSAVA2008\&PID $=24059 \&$ $\mathrm{O}=$ Generic). Accessed 21 November 2009.

96. Weese JS, et al. Cluster of methicillin-resistant Staphylococcus aureus colonization in a small animal intensive care unit. Journal of the American Veterinary Medical Association 2007; 231: 13611364.

97. NMC 2009. The National Mastitis Council: A practical look at contagious mastitis (http://nmconline.org/ contmast.htm). Accessed 26 October 2009.

98. Collignon P, et al. World Health Organization ranking of antimicrobials according to their importance in human medicine: a critical step for developing risk management strategies for the use of antimicrobials in food production animals. Clinical Infectious Diseases 2009; 49: 132-141.

99. BSAVA. BSAVA 2007: British Small Animal Veterinary Association, 2007. MRSA practice guidelines (http://www.bsava.com/Advice/MRSA/tabid/171/ Default.aspx). Accessed 21 November 2009.

100. Weese JS, et al. Isolation of methicillin-resistant Staphylococcus aureus from the environment in a veterinary teaching hospital. Journal of Veterinary Internal Medicine 2004; 18: 468-470.

101. Barton M, Hawkes M, Moore D. Guidelines for the prevention and management of community-associated methicillin-resistant Staphylococcus aureus: a perspective for Canadian health care practitioners. 2006 (http://www.ammi.ca/pdf/CA-MRSAFINAL_Jul19. pdf). Accessed 23 November 2009.

102. Coia JE, et al. Guidelines for the control and prevention of meticillin-resistant Staphylococcus aureus (MRSA) in healthcare facilities. Journal of Hospital Infection 2006; 63 (Suppl. 1): S1-44.

103. Ulvatne H. Antimicrobial peptides: potential use in skin infections. American Journal of Clinical Dermatology 2003; 4: 591-595.

104. van Duijkeren E, et al. Methicillin resistant Staphylococcus aureus (MRSA) infection in a dog in the Netherlands [in Dutch]. Tijdschrift voor diergeneeskunde 2003; 128: 314-315.

105. Weese JS. Methicillin-resistant Staphylococcus aureus: an emerging pathogen in small animals. Journal of the American Animal Hospital Association 2005; 41: $150-157$.

106. Rich M, Deighton L, Roberts L. Clindamycinresistance in methicillin-resistant Staphylococcus aureus 
isolated from animals. Veterinary Microbiology 2005; 111: 237-240.

107. Moreillon P. New and emerging treatment of Staphylococcus aureus infections in the hospital setting. Clinical Microbiology and Infection 2008; 14 (Suppl. 3): 32-41.
108. Enoch DA, et al. MRSA carriage in a pet therapy dog. Journal of Hospital Infection 2005; 60: 186-188.

109. Wright JG, et al. Infection control practices and zoonotic disease risks among veterinarians in the United States. Journal of the American Veterinary Medical Association 2008; 232: 1863-1872. 